\title{
Estimation of the onset rate and the number of asymptomatic patients of COVID-19 from the proportion of untraceable patients
}

\section{Takashi Odagaki ${ }^{1,2 *}$}

1Kyushu University, Fukuoka, 819-0395, J apan. 2Research Institute for Science Education, Inc., Kyoto, 603-8346, J apan. *Corresponding author. Email: t.odagaki@kb4.so-net.ne.jp

A simple method is devised to estimate the onset rate of COVID-19 from the proportion of untraceable patients tested positive, which allows us to obtain the number of asymptomatic patients, the number of infectious patients and the effective reproduction number. The recent data in Tokyo indicate that there are about six times as many infectious patients in the city as the daily confirmed new cases. It is shown that a quarantine measure on non-symptomatic patients is critically important in controlling the pandemic.

\section{Introduction}

The pandemic COVID-19 is still prevalent all over the world despite of continuous efforts by governments to control it. Difficulty of the control lies in the fact that patients of COVID-19 take a route different from common epidemics. In common epidemics, infected individuals show symptoms and become infectious after an incubation period. Then they are treated and recover from the disease. In COVID-19, infected individuals become infectious before symptom-onset and asymptomatic patients who do not show any symptoms before their recovery are infectious. In order to formulate proper strategies in controlling COVID-19, it is important to know the proportion of asymptomatic and pre-symptomatic patients (1), whom I call non-symptomatic patients collectively. However, it is unpractical to identify all non-symptomatic patients in the entire population by PCR tests. Therefore, it is an important problem to devise a method for estimating the number of infectious patients from data reported daily such as the confirmed new cases and the proportion of untraceable patients tested positive.

In this paper, I propose a simple method by which the onset rate $x$ of COVID-19 patients can be estimated from the proportion $f$ of untraceable patients tested positive and show that the proportion of the infectious patients can be obtained from the onset rate. I first analyze the infection process on the basis of the SIQR model (2) and find a relation between $x$ and $f$. Then, I argue that the number of infectious patients and the number of new patients on a given day can be related to the proportion $f$. I also discuss the effective reproduction number which depends on a quarantine rate of non-symptomatic patients and show that the quarantine of non-symptomatic patients is critically important in controlling COVID-19. I analyze the situation in Tokyo and discuss why COVID-19 does not converge in Tokyo.

\section{Classification of infectious patients}

I classify infected individuals on the basis of the SIQR model (2) and the mean field approach as follows. I first 
medRxiv preprint doi: https://doi.org/10.1101/2021.07.28.21261241; this version posted July 30, 2021. The copyright holder for this preprint (which was not certified by peer review) is the author/funder, who has granted medRxiv a license to display the preprint in perpetuity.

It is made available under a CC-BY-NC-ND 4.0 International license .

assume that patients of COVID-19 follow the same disease progression day by day at the same pace on average since their infection. On a particular day which I call day zero, there are many infected individuals who can be classified by the number of days since their infection. I denote by $x n_{0}$ the number of infected symptomatic individuals who are identified as patients by PCR tests and quarantined on day zero. Here, $x$ is the average onset rate of COVID-19, and therefore there are $(1-x) n_{0}$ asymptomatic patients who got infected on the same day as the quarantined patients got infected. I denote by $n_{-1}, n_{-2}, \cdots, n_{-d_{0}}$ the number of infected individuals who got infected one, two, $\cdots, d_{0}$ days later than the day when the $n_{0}$ individuals got infected. Similarly, I denote by $n_{1}, n_{2}, \cdots, n_{d_{1}-1}$ the number of individuals who got infected one, two, $\cdots, d_{1}-1$ days earlier than the day when the $n_{0}$ individuals got infected (3). Here, $d_{0}$ is the sum of the infectious period before symptom-onset and the period between the onset and getting quarantined, and $d_{1}$ denotes the infectious period of asymptomatic patients after symptomatic patients in the same group are quarantined. I assume that the proportion $1-x$ of asymptomatic patients is common in every group of patients. Figure 1 shows schematically the breakdown of patients into these three groups, where patients in the shaded area are infectious. Note that in the present analysis, the latent and incubation periods do not play any roles.

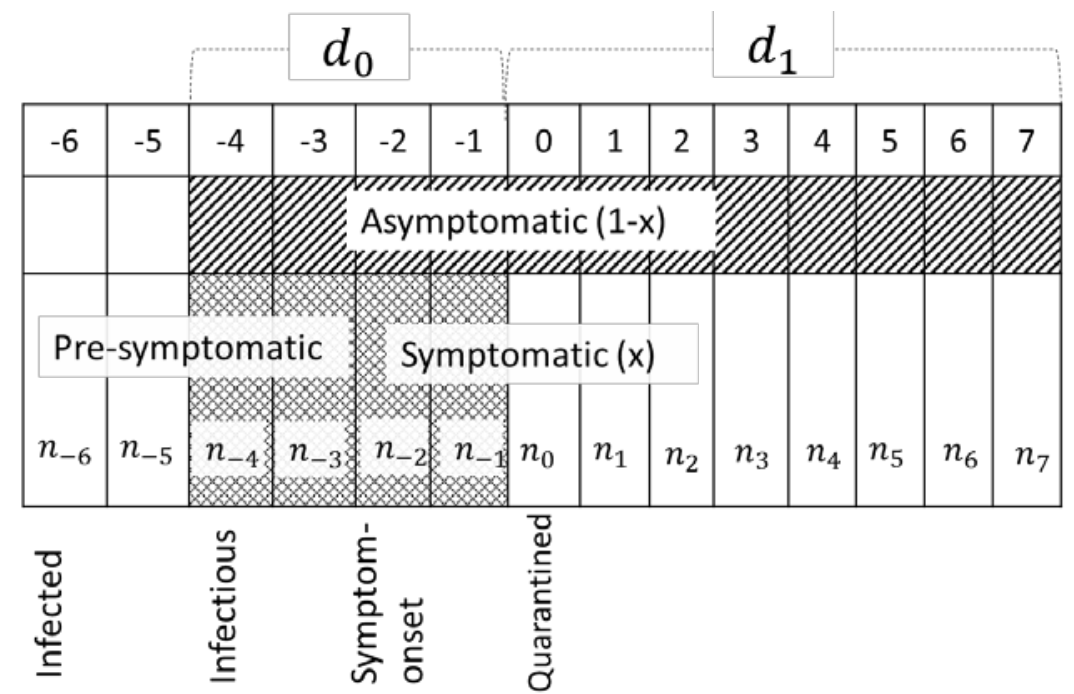

Fig.1. Break down of COVID-19 patients into asymptomatic, pre-symptomatic and symptomatic patients. Patients in the shaded area are infectious. The hatched region represents infectious asymptomatic patients who produce untraceable patients and the cross-hatched region corresponds to infectious pre-symptomatic and symptomatic patients who produce traceable patients.

I assume that all symptomatic patients will be tested intentionally some days after the symptom-onset and quarantined. I also assume that PCR tests are conducted on the general population and denote the quarantine rate of patients as $q$. If the quarantine measure on infected individuals is taken effectively, these infected patients decrease by a factor $(1-q)$ every day. Therefore, the number of infectious patients $\mathfrak{J}$ on day zero is given by

$$
\widetilde{J}=(1-q)^{T}\left\{\sum_{i=1}^{d_{0}} n_{-i}(1-q)^{-i}+(1-x) \sum_{i=0}^{d_{1}-1} n_{i}(1-q)^{i}\right\},
$$


medRxiv preprint doi: https://doi.org/10.1101/2021.07.28.21261241; this version posted July 30, 2021. The copyright holder for this preprint (which was not certified by peer review) is the author/funder, who has granted medRxiv a license to display the preprint in perpetuity.

It is made available under a CC-BY-NC-ND 4.0 International license.

and the new patients $\Delta I$ infected on day zero who will be identified some days later are given by

$$
\Delta I=(1-\mu)(1-a)(1-q)^{T}\left\{\sum_{i=1}^{d_{0}} \beta_{-i} n_{-i}(1-q)^{-i}+(1-x) \sum_{i=0}^{d_{1}-1} \beta_{i} n_{i}(1-q)^{i}\right\},
$$

where $T$ is the period that PCR test is effective before day zero, $\beta_{k}$ is the infection coefficient of patient group $n_{k}$, $\mu$ is the fraction of immunized individuals who are no longer susceptible, and $a$ represents the reduction rate of social contacts among people due to lockdown measures.

In order to make the following description transparent, I define an average of $n_{k}$ and a weighted average of $\beta_{k}$ as follows:

$$
\begin{aligned}
\langle n\rangle_{\mathrm{o}} & =\frac{1}{d_{0}} \sum_{i=1}^{d_{0}} n_{-i}(1-q)^{-i}, \\
\langle n\rangle_{1} & =\frac{1}{d_{1}} \sum_{i=0}^{d_{1}-1} n_{i}(1-q)^{i}, \\
\langle\beta\rangle_{\mathrm{o}} & =\sum_{i=1}^{d_{0}} \beta_{-i} n_{-i}(1-q)^{-i} / \sum_{i=1}^{d_{0}} n_{-i}(1-q)^{-i}, \\
\langle\beta\rangle_{1} & =\sum_{i=0}^{d_{1}-1} \beta_{i} n_{i}(1-q)^{i} / \sum_{i=0}^{d_{1}-1} n_{i}(1-q)^{i},
\end{aligned}
$$

and write Eqs. (1) and (2) as

$$
\mathfrak{J}=(1-q)^{T}\left\{d_{0}\langle n\rangle_{0}+(1-x) d_{1}\langle n\rangle_{1}\right\}
$$

and

$$
\Delta I=(1-\mu)(1-a)(1-q)^{T}\left\{d_{0}\langle\beta\rangle_{0}\langle n\rangle_{0}+(1-x) d_{1}\langle\beta\rangle_{1}\langle n\rangle_{1}\right\}
$$

\section{Relation between the proportion of untraceable patients and the onset rate}

Out of the newly infected individuals $\Delta I, \Delta Q \equiv x \Delta I$ will show symptoms some days later and $(1-x) \Delta I$ will not show any symptoms. Patients $\Delta Q$ showing symptoms will be identified by PCR tests and be listed as daily confirmed new cases. I assume that they are classified into two groups $\Delta Q_{t}$ and $\Delta Q_{u n t}$, where $\Delta Q_{t}$ are traceable patients who are infected from symptomatic and pre-symptomatic patients and $\Delta Q_{u n t}$ are untraceable patients who are infected from asymptomatic patients. Namely, $\Delta Q_{t}$ and $\Delta Q_{u n t}$ are given by

$$
\begin{aligned}
\Delta Q_{t} & =(1-\mu)(1-a)(1-q)^{T} x^{2} d_{0}\langle\beta\rangle_{0}\langle n\rangle_{0}, \\
\Delta Q_{u n t} & =(1-\mu)(1-a)(1-q)^{T} x(1-x)\left\{d_{0}\langle\beta\rangle_{0}\langle n\rangle_{0}+d_{1}\langle\beta\rangle_{1}\langle n\rangle_{1}\right\} .
\end{aligned}
$$

Therefore, the proportion of untraceable cases in the daily confirmed new cases is given by

$$
f \equiv \frac{\Delta Q_{u n t}}{\Delta Q}=\frac{(1-x)\left[d_{0}\langle\beta\rangle_{0}\langle n\rangle_{0}+d_{1}\langle\beta\rangle_{1}\langle n\rangle_{1}\right]}{d_{0}\langle\beta\rangle_{0}\langle n\rangle_{0}+(1-\mathrm{x}) d_{1}\langle\beta\rangle_{1}\langle n\rangle_{1}}
$$


medRxiv preprint doi: https://doi.org/10.1101/2021.07.28.21261241; this version posted July 30, 2021. The copyright holder for this preprint (which was not certified by peer review) is the author/funder, who has granted medRxiv a license to display the preprint in perpetuity.

which does not depend on $\mu, a$ and $T$ explicitly. This relation can be inverted to get

$$
x=\frac{1-f}{1-\frac{d_{1}\langle\beta\rangle_{1}\langle n\rangle_{1}}{d_{0}\langle\beta\rangle_{0}\langle n\rangle_{0}+d_{1}\langle\beta\rangle_{1}\langle n\rangle_{1}} f},
$$

which indicates that the onset rate $x$ can be obtained from the proportion of untraceable patients $f$ once other parameters are known.

In order to incorporate the trend of infection status (4) into the present analysis, I first define trend parameters $\tau_{0}$ and $\tau_{1}$ by $\tau_{0}=\langle n\rangle_{0} / n_{0}$ and $\tau_{1}=\langle n\rangle_{1} / n_{0}$. It is apparent that the infection status after day zero will be increasing, stationary and decreasing when $\tau_{0}>1, \tau_{0}=1$ and $\tau_{0}<1$, respectively. Similarly, $\tau_{1}>1, \tau_{1}=1$ and $\tau_{1}<1$ indicate that the infection status before day zero has been increasing, stationary and decreasing, respectively. It is straight forward to express $x$ in terms of $\tau_{0}$ and $\tau_{1}$ as

$$
x=\frac{1-f}{1-\frac{d_{1}\langle\beta\rangle_{1} \tau_{1}}{d_{0}\langle\beta\rangle_{0} \tau_{0}+d_{1}\langle\beta\rangle_{1} \tau_{1}} f} .
$$

Therefore, $\Delta I$ is written as

$$
\Delta I=(1-\mu)(1-a)(1-q)^{T}\left\{d_{0}\langle\beta\rangle_{0} \tau_{0}+\frac{\langle\beta\rangle_{0}\langle\beta\rangle_{1} d_{0} \tau_{0} d_{1} \tau_{1}}{d_{0}\langle\beta\rangle_{0} \tau_{0}+d_{1}\langle\beta\rangle_{1} \tau_{1}(1-f)} f\right\} n_{0} .
$$

Since the confirmed new cases on day zero $(\Delta Q)_{0}$ is given by $(\Delta Q)_{0}=x n_{0}, \mathfrak{J}$ can be expressed in terms of $\tau_{0}$, $\tau_{1}$ and $(\Delta Q)_{0}$ as

$$
\mathfrak{J}=\frac{(1-q)^{T}}{1-f}\left[d_{0} \tau_{0}+\frac{\left(\langle\beta\rangle_{0}-\langle\beta\rangle_{1}\right) d_{0} \tau_{0} d_{1} \tau_{1}}{d_{0}\langle\beta\rangle_{0} \tau_{0}+d_{1}\langle\beta\rangle_{1} \tau_{1}} f\right](\Delta \mathrm{Q})_{0}
$$

\section{Number of infectious patients and asymptomatic patients in Tokyo}

Now, I apply the present analysis to the infection status in Tokyo. In Tokyo, PCR tests have not conducted on non-symptomatic patients and I can set $q=0$. I first assume that the infection status was stationary as in middle of June, 2021, and set $\tau_{0}=\tau_{1}=1$ and $\langle\beta\rangle_{0}=\langle\beta\rangle_{1}$. Then, Eq. (13) reduces to

$$
x=\frac{1-f}{1-\frac{d_{1}}{d_{0}+d_{1}} f} .
$$

According to Ministry of Health, Labour and Welfare of Japan (MHLW), transmission occurs in the 2 days before symptom-onset and in the 7 10 days post symptom-onset (5). These estimations are consistent with those reported in Ontario, Canada: Ontario Agency for Health Protection and Promotion reported that the transmission occurs in the $3 \sim 5$ days before onset and in the 8 10 days post onset (3). Assuming on average a patient will be tested and quarantined next day of symptom-onset, I set as a model case $d_{0}=3$ days and $d_{1}=7$ days. Then, Eq. (16) is reducible to

$$
x=\frac{1-f}{1-\frac{7}{10} f}
$$


medRxiv preprint doi: https://doi.org/10.1101/2021.07.28.21261241; this version posted July 30, 2021. The copyright holder for this preprint (which was not certified by peer review) is the author/funder, who has granted medRxiv a license to display the preprint in perpetuity.

It is made available under a CC-BY-NC-ND 4.0 International license .

which is shown in Fig.2 In Tokyo, the proportion of untraceable patients is $f=50 \sim 60 \%$. Using the lower value $f$ $=50 \%$, I find $x=77 \%$. This value becomes $x=69 \%$ if $f=60 \%$ is used. These values are consistent with observations of the onset rate $x=76 \%$ (7) or $75 \%$ (8).

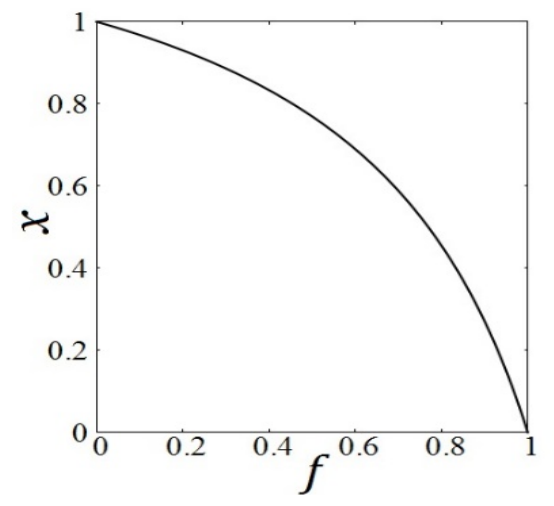

Fig. 2. $x$ vs $f$ when $d_{0}=3$ days and $d_{1}=7$ days.

On the same conditions, the number of infectious patients Eq. (15) can be written as

$$
\frac{\mathfrak{I}}{(\Delta Q)_{0}}=\frac{d_{0}}{1-f} \text {. }
$$

If there were no untraceable patients, all infected individuals would be symptomatic and, therefore, the number of infectious patients will be given by the number of new cases times days during which they are infectious, i.e. $\mathfrak{J} /$ $(\Delta Q)_{0}=d_{0}$ when $f=0$ as Eq. (18) indicates. Equation (18) shows $\mathfrak{J} /(\Delta Q)_{0}=2 d_{0}$ and $2.5 d_{0}$ when $f=$ $50 \%$ and $60 \%$ respectively. Figure 3 shows $f$ dependence of $\mathfrak{J} /(\Delta Q)_{0}$ when $d_{0}=3$ days and $d_{1}=7$ days. It is important to note that in Tokyo $f=50 \sim 60 \%$ and $d_{0}=3$ days, and thus there are $6 \sim 7.5$ times more

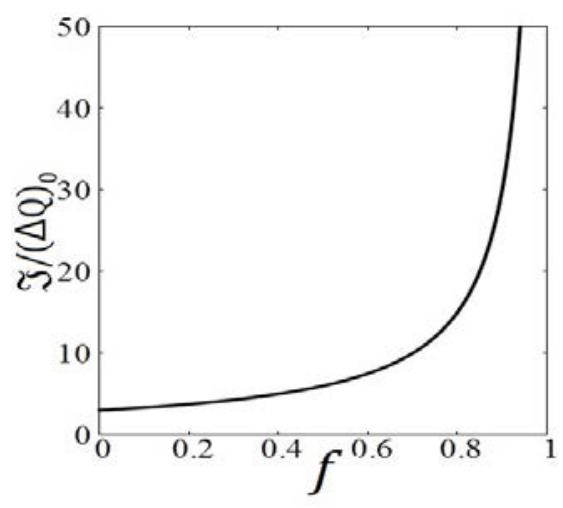

Fig. 3. Dependence of $\mathfrak{J} /(\Delta Q)_{0}$ on $f$ when $d_{0}=3$ days and $d_{1}=7$ days.

infectious patients than the daily confirmed new cases. It should also be mentioned that the number of asymptomatic infectious patients $\mathfrak{\Im}_{\text {as }}$ excluding pre-symptomatic patients is given by

$$
\frac{\widetilde{I}_{\mathrm{as}}}{(\Delta Q)_{0}}=\frac{f d_{0}}{1-f}
$$

and thus $\mathfrak{I}_{\text {as }} /(\Delta Q)_{0}=3$ when $d_{0}=3$ days, $d_{1}=7$ days and $f=50 \%$. 
medRxiv preprint doi: https://doi.org/10.1101/2021.07.28.21261241; this version posted July 30, 2021 . The copyright holder for this preprint (which was not certified by peer review) is the author/funder, who has granted medRxiv a license to display the preprint in perpetuity.

It is made available under a CC-BY-NC-ND 4.0 International license .

\section{Effective reproduction number and assessment of policies}

I first define an effective infectious period $d_{\text {eff }}$ and an effective infection coefficient $\beta_{\text {eff }}$ as follows:

$$
\begin{aligned}
& d_{\text {eff }}=(1-q)^{T}\left\{d_{0} \tau_{0}+(1-x) d_{1} \tau_{1}\right\}, \\
& \beta_{\text {eff }}=\frac{\langle\beta\rangle_{0} d_{0} \tau_{0}+(1-x)\langle\beta\rangle_{1} d_{1} \tau_{1}}{d_{0} \tau_{0}+(1-x) d_{1} \tau_{1}} .
\end{aligned}
$$

Equation (8) can now be expressed as

$$
\Delta I=(1-\mu)(1-a) \beta_{\mathrm{eff}} d_{\mathrm{eff}} n_{0},
$$

and the effective reproduction number $R_{\text {eff }}$ on day zero is given by

$$
R_{\mathrm{eff}} \equiv \frac{\Delta I}{n_{0}}=(1-\mu)(1-a) \beta_{\mathrm{eff}} d_{\mathrm{eff}}
$$

It should be remarked that when $d_{\text {eff }}$ is equal to the recovery time or the inverse of the recovery rate $\gamma$ and $\beta_{\text {eff }}$ is a constant, the expression for $R_{\text {eff }}$ is identical to that defined in the standard SIR model. It is important to note that, in contrast to the standard SIR model, the effective reproduction number $R_{\text {eff }}$ defined by Eq. (23) depends on the quarantine rate of non-symptomatic patients through $d_{\text {eff }}$.

The basic strategy against COVID-19 is to bring the effective reproduction number smaller than unity so that the number of patients decreases. Equation (23) indicates that there are three tactics: (1) increase immunized people by vaccination or by natural immunity to make $(1-\mu)$ smaller, (2) enforce social distancing by various lockdown measures to make $(1-a)$ smaller, and (3) quarantine asymptomatic patients by PCR tests to make $(1-q)$ or $d_{\text {eff }}$ smaller. It is important to note that quarantine of non-symptomatic patients reduces the effective reproduction number by $(1-q)^{T}$ which could be significant because of exponent $T$ if $q$ is not negligible. In countries like Taiwan, Australia and New Zeeland who have succeeded in controlling COVID-19 before the vaccination was started, PCR tests have been conducted more than 100 times per positive patient in a well-designed manner.

In Tokyo, the PCR test has been used only to confirm the infection of novel corona virus for people who show some symptoms, which means $q=0$, and thus it has not been contributing to the battle against COVID-19. Furthermore, lockdown measures have been very sloppy. Instead of enforcing social distancing among the entire population it has been applied only specific targets, like the night life district last year and restaurants serving alcohol beverage this year. The policy targeting on certain shops and opening hours has only limited effects on social distancing since people gather together in a park or on a street. Furthermore, the policy has been enforced and lifted every one or two months, which has caused the wavy infection curve $(9,10)$. It could be possible to increase $a$ by, for example, promoting Telework, limiting working days, reducing crowd in commuter trains and banning gatherings.

\section{Discussion and conclusion}

In the present analysis, traceable and untraceable patients tested positive are related to infection from pre-symptomatic and symptomatic patients and from asymptomatic patients, respectively. Although this assumption may not be rigorous, it is a good assumption if people cooperate in the investigation of infection route by the health 
medRxiv preprint doi: https://doi.org/10.1101/2021.07.28.21261241; this version posted July 30, 2021. The copyright holder for this preprint (which was not certified by peer review) is the author/funder, who has granted medRxiv a license to display the preprint in perpetuity.

It is made available under a CC-BY-NC-ND 4.0 International license .

department of local government. It should also be mentioned that, since the important point is if patients are quarantined or not, the present results do not depend on the accuracy of PCR test at all.

In the analysis of asymptomatic patients in Tokyo, I assumed that the infection status is stationary. The infection status can also be increasing or decreasing (4). However, if the condition $\langle\beta\rangle_{0}=\langle\beta\rangle_{1}$ is satisfied, the relation Eq. (15) between the number of infectious patients and the number of the newly confirmed new cases reduces to

$$
\frac{\mathfrak{I}}{(\Delta \mathrm{Q})_{0}}=\frac{(1-q)^{T}}{1-f} d_{0} \tau_{0}
$$

which depends only on $d_{0} \tau_{0}$ when $q=0$. This ratio becomes large in the increasing status and small in the decreasing status compared to Eq. (18). In this case, the effective reproduction number depends on the infection status through $d_{\text {eff }}$. In general, $d_{\text {eff }}$ is an increasing function of $\tau_{0}$ and $\tau_{1}$. Since the increasing state corresponds to $\tau_{0}>1>\tau_{1}$ and the decreasing state to $\tau_{0}<1<\tau_{1}, d_{\text {eff }}$ can increase or decrease depending on the relation between $\tau_{0}$ and $\tau_{1}$.

The infection coefficient of SARS-CoV-2 depends on variants. When a new variant with stronger infection coefficient emerges, $\langle\beta\rangle_{0}$ becomes larger than $\langle\beta\rangle_{1}$. The number of infectious patients will increases for a stronger variant when other parameters are the same.

In 2021, vaccination has been progressed in many countries and the infection status seems to be improving at least in the reduction of serious cases. The effect of vaccination appears through $\mu$ in Eq. (2) which depends on variants of the virus. Therefore policies for controlling COVID-19 should not rely only on the vaccination, and a proper combination of policies on vaccination, social distancing and quarantine of non-symptomatic patients must be designed in each country around the world.

I have shown in this paper that important information on the onset rate and the number of infectious patients can be estimated from the proportion of untraceable patients tested positive. The effective reproduction number depends not only on the vaccination rate and effects of social distancing but also on $d_{\text {eff }}$ which is controlled by quarantine measures on pre-symptomatic and asymptomatic patients. The effect of the quarantine measure appears as reduction of the effective reproduction number by $(1-q)^{T}$ which could be significantly large compared to the effects of the lockdown measures and the vaccination.

\section{REFERENCES}

1. R. Subramanian, Q. He, and M. Pascual, Quantifying asymptomatic infection and transmission of COVID-19 in New York City using observed cases, serology, and testing capacity, Proc. Nat. Acad. Sci., 118, e2019716118 (2021). https://doi.org/10.1073/pnas.2019716118

2. T. Odagaki, Analysis of the outbreak of COVID-19 in Japan by SIQR model, Infectious Disease Modelling, $\mathbf{5}$, 691-698 (2020). https://doi.org/10.1016/.idm.2020.08.013

3. Ontario Agency for Health Protection and Promotion (Public Health Ontario). COVID-19 overview of the period of communicability - what we know so far. Toronto, ON: Queen's Printer for Ontario; 2021. https://www. publichealthontario.ca/-/media/documents/ncov/covid-wwksf/2021/03/wwksf-period-of-communic ability-overview.pdf?la=en 
medRxiv preprint doi: https://doi.org/10.1101/2021.07.28.21261241; this version posted July 30, 2021. The copyright holder for this preprint (which was not certified by peer review) is the author/funder, who has granted medRxiv a license to display the preprint in perpetuity. It is made available under a CC-BY-NC-ND 4.0 International license.

4. T. Odagaki and R. Suda, Classification of the infection status of COVID-19 in 190 countries, J. Clinical Trials to be published (2021). https://doi.org/10.1101/2020.12.17.20248445

5. Ministry of Labour, Health and Welfare, Japan, "Guidance for treatment of COVID-19" ver5.1, July, 5, (2021). https://www.mhlw.go.jp/content/000801626.pdf

6. Tokyo Metropolitan Government, "Updates on COVID-19 in Tokyo", July 20 (2021)

https://www.fukushihoken.metro.tokyo.lg.jp/iryo/kansen/corona portal/info/monitoring.images/0715graph3-1 . $\mathrm{JPG}$

7. M. A. Johansson, T. M. Quandelacy, S. Kada, P. V. Prasad, M. Steele et al, SARS-CoV-2 Transmission From People Without COVID-19 Symptoms, JAMA Network Open. 4(1) e2035057 (2021).

http://doi:10.1001/jamanetworkopen.2020.35057

8. M. Alene, L. Yismaw, M. A. Assemie. D. B. Ketema, B. Mengist et al, Magnitude of asymptomatic COVID-19 cases throughout the course of infection: A systematic review and meta-analysis, PLoS ONE 16(3), e0249090 (2021). https://doi.org/10.1371/journal.pone.0249090

9. T. Odagaki, Self-organized wavy infection curve of COVID-19, Scientific Reports, 11, 1936 (2021). https://doi.org/10.1038/s41598-021-81521-z

10. T. Odagaki, Self-organization of oscillation in an epidemic model for COVID-19, Physica A 573, 125925 (2021). https://doi.org/10.1016/i.physa.2021.125925

\section{ACKOWLEDGMENTS}

This work was supported in part by JSPS KAKENHI Grant Number 18 K03573. 